Apidologie, 1981, 12 (4), 383-396.

\title{
SÉCRÉTIONS ET BIOLOGIE FLORALE. I. NATURE, ORIGINE ET RÔLE DES SÉCRETTIONS DANS LA POLLINISATION ET LA FÉCONDATION : REVUE DES DONNÉES RÉCENTES
}

\author{
Pierre ZANDONELLA, Christian DUMAS et Thierry GAUDE \\ Histophysiologie de la Sécrétion, 43, boulevard du 11-Novembre 1918, Lyon I, F-69622 Villeurbanne \\ Cedex
}

\section{RÉSUMÉ}

Au cours des dix dernières années, les aspects morphologiques liés à la biologie florale ont été complétés par des données concernant des aspects plus dynamiques. Les rôles des sécrétions dans la pollinisation et la fécondation ont été précisés et le mécanisme de ces sécrétions partiellement élucidé.

Cette mise au point résume les données récentes concernant les nectars, les odeurs, les sécrétions polliniques, tapétales et pistillaires.

La composition des nectars, la structure des nectaires, les processus de sécrétion sont très largement abordés.

La production d'odeurs attractives pour les pollinisateurs par les cellules du périanthe ou par des osmophores est également envisagée. Les substances émises sont essentiellement des terpènes et des amines.

Les stigmates, de type humide ou sec, et les exsudats stigmatiques sont évoqués tant pour leur rôle dans la pollinisation que dans la fécondation. Les autres sécrétions pistillaires liées à la fécondation sont également citées.

Enfin, les grains de pollen véhiculés par les pollinisateurs sont entourés d'un manteau (" pollen coat ") qui intervient dans les mécanismes d'adhésion pollen-pollen et pollen-pistil.

Des sécrétions spécifiques font partie des processus qui concourent à assurer la fécondation croisée chez les Angiospermes. Elles sont de deux types. Les unes interviennent au cours de la pollinisation, transport du pollen, de l'étamine au stigmate. Ce sont les nectars, les odeurs qui associées aux couleurs contribuent à attirer l'animal vecteur du pollen. On ne les rencontre généralement que chez les espèces zoogames. Les autres jouent un rôle essentiel dans la seconde phase que l'on peut qualifier de fécondation, comprenant l'adhésion du pollen sur le stigmate et les mécanismes qui aboutissent à la syngamie. Elles se situent au niveau des grains de pollen et de divers tissus du pistil.

Plusieurs ouvrages généraux récents, parmi lesquels nous retiendrons ceux de FAEGRı et Van der PIJL (1971) et de Frankel et GaLun (1977), témoignent d'un regain d'intérêt pour la biologie florale. Si les 
structures impliquées dans la pollinisation ont fait l'objet de multiples études déjà anciennes, la connaissance de la biochimie des substances sécrétées (voir HARBORNE, 1977) et de la cytologie des glandes (voir FAHN, 1979 b) est d'acquisition récente. Les divers processus impliqués dans la phase de fécondation ne sont que depuis peu l'objet de recherches approfondies. Ils s'avèrent complexes mais d'un intérêt insoupçonné.

La présente mise au point ne concerne que les données les plus récentes et ne fait référence qu'aux travaux des dix dernières années, sauf dans le cas où ils manquent. De nombreux travaux antérieurs sont fondamentaux et sources des recherches actuelles. Cependant, leurs données bien connues, car de nombreuses fois analysées, ne seront pas reprises ici.

\section{LES NECTARS}

Ce sont des solutions sucrées sécrétées par des glandes que l'on peut regrouper sous le terme général de nectaires.

a) Composition

Des analyses nombreuses ont été publiées plus ou moins complètes et précises selon les techniques utilisées. Les compositions sont complexes dans le détail. Nous en référons à l'analyse bibliographique de FAHN (1979 b).

Nous en retiendrons ce qui peut être généralisé. Cela concerne surtout les sucres qui constituent l'essentiel de la matière sèche des nectars. Le saccharose, le glucose et le fructose dominent, ces deux derniers sucres sont toujours associés et en quantités équilibrées. Leurs proportions relatives sont connues chez plus d'un millier d'espèces et très variées. La plupart des nectars sont des mélanges des trois sucres, mais certains ne possèdent que du saccharose d'autres que du glucose-fructose. Différents types peuvent coexister chez des espèces très voisines, comme HaRborne (1977) le signale chez Rhododendron. Outre ces sucres dominants, de nombreux oses ont été identifiés, au moins sous forme de traces. La chromatographie en phase gazeuse permet depuis peu de révéler la complexité de certains nectars par rapport à d'autres (12 sucres chez Epipactis purpurea: Pais et Chaves Das Neves, 1980; saccharose seul chez Thunbergia laurifolia: Zandonella et Piolat, 1981).

Des protéines, des acides aminés, des enzymes, des lipides, des acides organiques, des phosphates, des ions minéraux entrent également dans la composition des nectars. BAKER et BAKER ont, au cours de ces dernières années, identifié les acides aminés de nombreux nectars. Outre l'intérêt taxonomique, la composition en acides aminés étant spécifique, ces auteurs ont montré que les nectars les plus riches en acides aminés sont produits par les fleurs pollinisées par les animaux se nourrissant exclusivement de nectar (voir BAKER, 1977). Les acides aminés, ainsi d'ailleurs que des cations - comme le potassium - à la présence desquels on commence à s'intéresser, contribuent à la sapiditẻ du nectar et lui confèrent des qualités attractives ou répulsives. Il est curieux de noter qu'occasionnellement on a détecté la présence de substances toxiques naturelles. Par ailleurs, on retrouve dans le nectar des concentrations non négligeables de pesticides plusieurs jours après traitement, en particulier par les systémiques comme le montrent de nombreux travaux réalisés aux États-Unis depuis une quinzaine d'années (voir BARKER et al., 1980).

Des nectars peuvent être riches en lipides (BAKER, 1977). Dans certains cas, des lipides seuls constituent l'appât (Vogel, 1974).

\section{b) Les nectaires}

Des nectars peuvent être riches en lipides (BARKER, 1977). Dans certains cas, des lipides seuls constinectaires floraux ou de nectaires extrafloraux, ou, selon leur fonction, de nectaires nuptiaux ou de nectaires extranuptiaux.

La notion de nectaires nuptiaux moins habituelle que celle de nectaires floraux nous semble particulièrement convenir au sujet traité ici. Il n'est pas rare, en effet, de constater l'intervention de sécrétions extraflorales associées à des sécrétions florales dans le but d'assurer la visite de la fleur par le pollinisateur. L'exemple de Thunbergia grandiflora (VAN der PIJL, 1954) est particulièrement significatif. Des fourmis, appâtées par le nectar extrafloral, interdisent l'accès à l'extérieur de la corolle régulièrement percée en leur absence par des prédateurs. Les insectes doivent alors accéder au nectar floral par l'intérieur de la corolle 
et, ainsi, contactent les organes reproducteurs. Le rôle des nectars extrafloraux dans la protection de la plante par les fourmis, et, de la fleur en particulier, est généralement admis aujourd'hui (BENTLEY, 1977).

La structure des glandes à nectär est très variée. Certains sites de sécrétion, essentiellement extrafloraux, ne se distinguent par aucune structure histologique en dehors d'une vascularisation et de stomates. Mais, la plupart des nectaires sont structurés et constitués de cellules à caractères glandulaires. Nous groupons ces derniers en deux types histologiques : un type épidermique comprenant les épithéliums et les trichomes, et un type parenchymateux constitué de nombreuses assises cellulaires (Fig. 1).
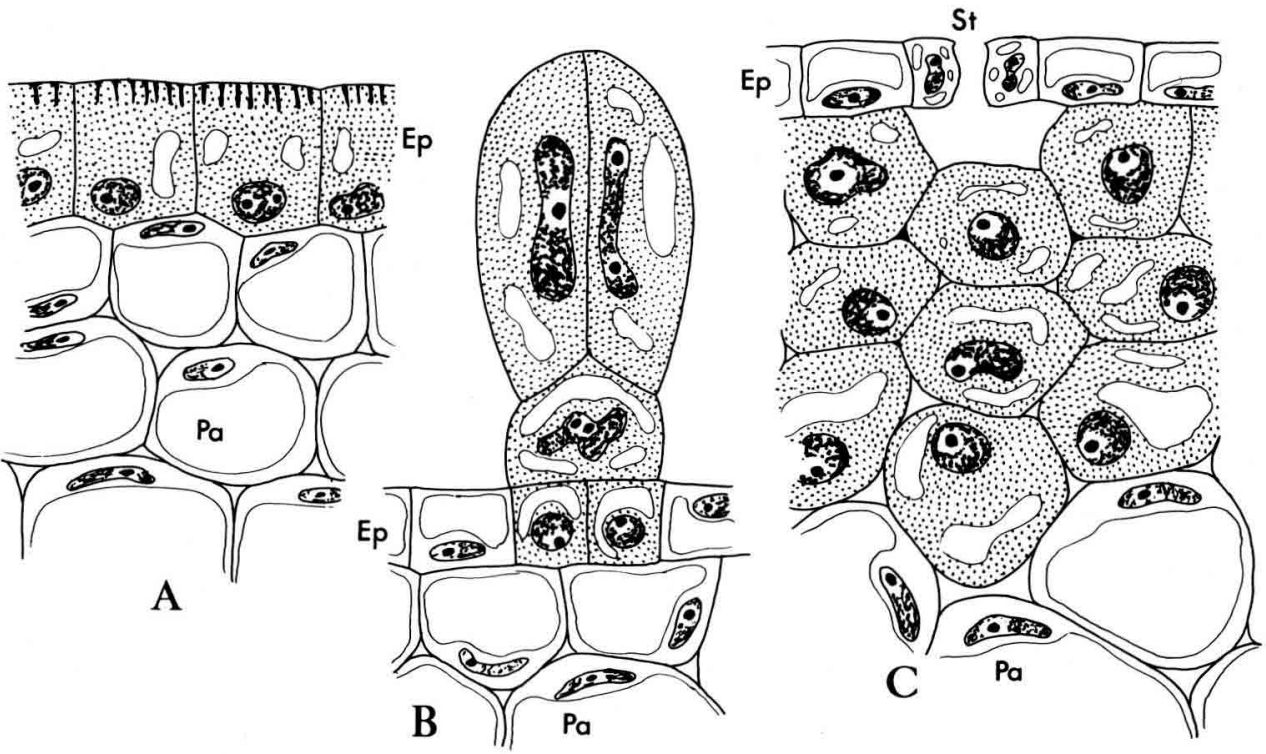

FIG. 1. - Types histologiques de nectaires structurés.

A : épithélium, B : trichome, C : parenchyme.

Le cytoplasme des cellules glandulaires est figuré en pointillés.

(Ep : épiderme, $\mathrm{Pa}$ : parenchyme banal, $\mathrm{St}$ : stomate.)

FIG. 1. - Histological types of structured nectaries.

A : epithelium; B : trichome; C : parenchyma.

Cytoplasma of gland cells are stippled

(Ep : epidermis; $\mathrm{Pa}$ : usual parenchyma; $\mathrm{St}$ : stoma).

\section{c) Modalités de la sécrétion}

Il est admis, d'une manière générale, que le nectar trouve sa source dans la sève élaborée. Cette conviction est basée plus particulièrement sur la connaissance de la composition de la sève et du nectar et sur la relation existant entre la composition du nectar et le type de vascularisation (phloème seul ou phloème et xylème) du nectaire. En ce domaine, tous les travaux récents font référence aux contributions fondamentales de Frey-Wissling, Frei, Agthe, Zimmermann, Ziegler, Lüttge, Kartashova, etc. (voir FAHN, 1979 b).

Mais, le problème de la sécrétion nectarifère n'est pas résolu pour autant, cette sécrétion n'étant pas, dans la plupart des cas une simple exsudation de sève. La composition des deux substances présente souvent des différences notables. La nature et le transit des produits issus de la sève élaborée, constituant le pronectar, les mécanismes et les sites de leur remaniement et de l'exsudation du nectar sont l'objet d'hypothèses basées en grande partie sur les données de l'étude cytologique et parfois cytochimique des cellules glandulaires. Là encore, dans le cadre de cette revue nous passerons sous silence les contributions très 
connues d'auteurs comme SCHNEPF, EymÉ, Figier, LÜTTGe, etc. (voir FAHN, 1979 b) pour ne citer que les plus récentes.

Le premier type d'informations concerne le trajet du pronectar qui peut emprunter la voie apoplastique ou la voie symplastique. Un transport symplastique est vraisemblable dans de nombreux cas comme l'indique par exemple l'abondance de plasmodesmes (Findlay et MerCer, 1971; Wergin et al., 1975; GunNing et Hughes, 1976). Cela est peu contestable chez les glandes de type épidermique (Fig. 2 a). Par contre, dans les parenchymes, à défaut d'indice net, on peut admettre, au moins pour une partie du pronectar, l'hypothèse de VASILIJEv (1969, 1971) d'un trajet apoplastique (Fig. 2).
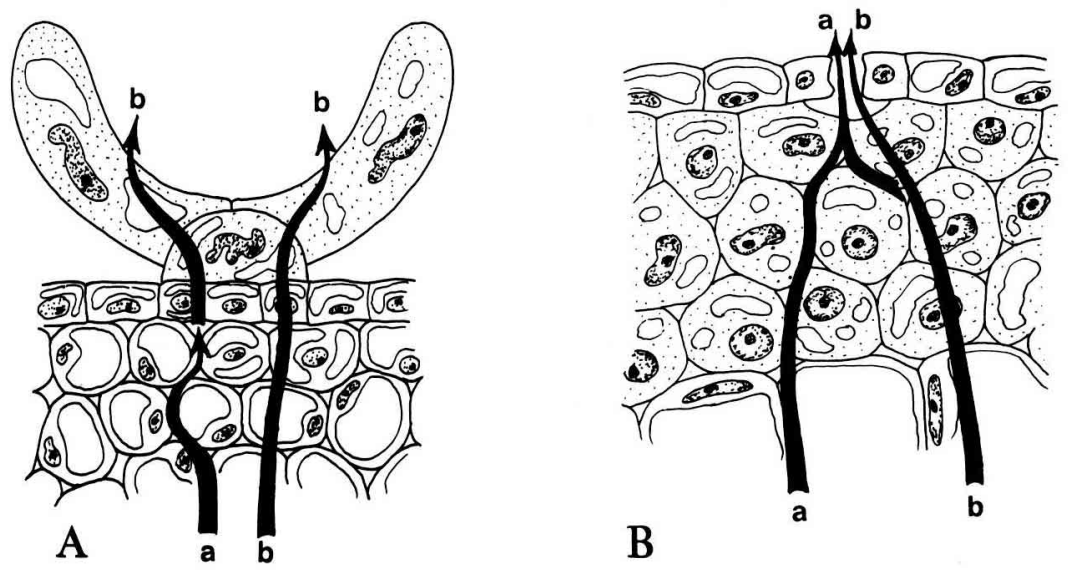

FIG. 2. - Trajets possibles du pronectar.

A : nectaire de type épidermique, B : nectaire de type parenchymateux,

a : trajet apoplastique, $b$ : trajet symplastique.

FIG. 2. - Possible ways of pronectar.

A : epidermis-type nectary; B : parenchyma-type nectary.

a : apoplastic way; b : symplastic way.

Le second type d'informations, lié au précédent, concerne les sites cellulaires de transit et de remaniement éventuel. Il convient de souligner qu'il manque, dans la plupart des cas, la connaissance préalable de la composition de la sève élaborée au voisinage du nectaire, du pronectar et même du nectar en raison de la difficulté de leur obtention. Les données récentes sont encore rares (Zauralov et Pavlinova, 1975; BAKER et al., 1978). Elles apportent de précieuses informations sur les types de remaniement du pronectar et permettent d'envisager avec plus de probabilité les sites de ces remaniements.

En raison de l'abondance fréquente du réticulum endoplasmique, FAHN et RAchmilevitz (1970, 1975 ) considèrent qu'il est le lieu de transit des précurseurs du nectar, ce que confirment plusieurs auteurs. La mise en évidence par HeINRICH (1975) de plusieurs hydrolases dans le réticulum apporte un argument de poids à cette thèse. L'activité sécrétrice du golgi, souvent intense, a été signalée par plusieurs auteurs dont récemment BENNER et SCHNEPF (1975). Une continuité est aujourd'hui envisagée entre le réticulum et le golgi (FAHN et BenoualChe, 1979; FAHN, 1979 a). Il s'agirait là d'un transit du nectar par une voie exocytaire qui semble fréquente dans la cellule. La sécrétion est de type granulocrine.

Mais la présence de tels indices est loin d'être générale, principalement dans les parenchymes (VASILL Jev, 1969, 1971; Zandonella, 1970, 1972; Zandonella et Piolat, 1981). Dans ce cas, la sécrétion peut être de type eccrine. Le rôle du plasmalemme est alors essentiel. LÜTTGE (1977) analyse les possibilités de transport membranaire des sucres et acides aminés. Dans de nombreux nectaires de type épidermique, la surface du plasmalemme est accrue de façon considérable par le développement de protubérances pariétales (voir SCHNepf et Pross, 1976; FaHN, 1979 a). 
L'intervention des plastes n'a guère été envisagée. Dans bien des cas, ils ne contiennent que peu d'amidon. Quand cet amidon est abondant, il n'est pas toujours utilisé au cours de la sécrétion (ÉRIKSSON, 1977), mais il peut l'être (Zandonella et PiOlat, 1981) et constituer la source probable d'une partie des sucres du nectar.

En conclusion, les processus sécrétoires sont diversifiés et complexes (LüTTGE et SCHNEPF, 1976; SCHNEPF, 1977; FAHN, 1979 a et b) (Fig. 3). Il est pensable que plusieurs d'entre eux interviennent dans l'élaboration d'un même nectar. Le fait que des structures notablement différentes puissent produire des nectars tout à fait voisins (ZANDONELla et PIOLAT, 1981) est. en tout cas, remarquable et devrait entrainer une nouvelle réflexion sur les rapports entre les structures et la sécrétion.

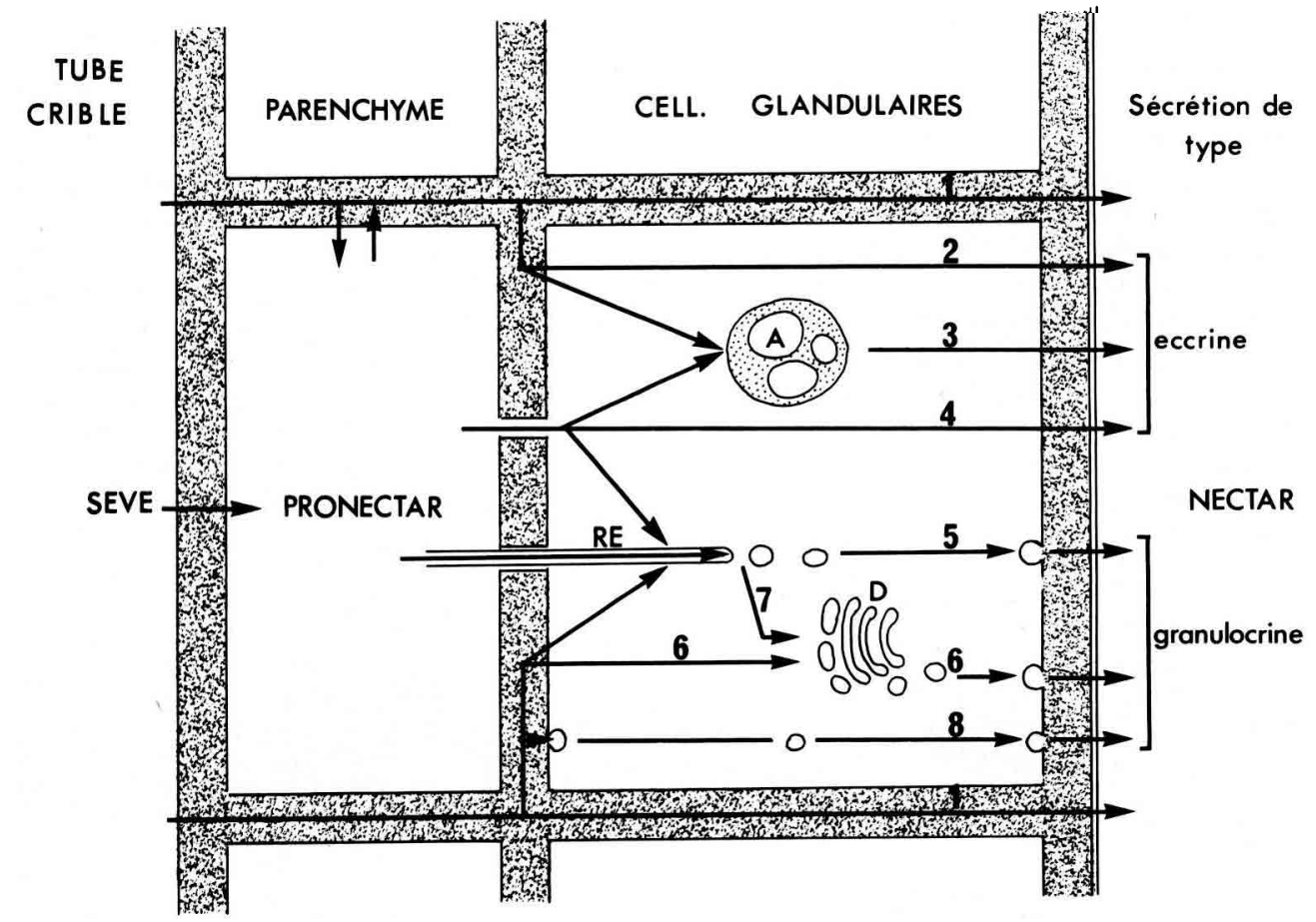

FIG. 3. - Trajet possible du pronectar et types de sécrétion (inspiré de ScHNEPF, 1977).

A : amyloplaste, $\mathrm{D}$ : dictyosome, RE : réticulum endoplasmique.

1 : trajet apoplastique; 2 à 4 : sécrétion eccrine avec éventuellement intervention des amyloplastes;

5 à 8 : sécrétion granulocrine impliquant le réticulum endoplasmique et le Golgi, seuls ou associés avec le plasmalemme.

FIG. 3. - Possible way of pronectar and secretion types (inspired from SCHNEPF, 1977).

A : amyloplast; D : dictyosome; RE : endoplasmic reticulum.

1 : apoplastic way; $2-4:$ eccrine secretion with possible intervening of amyloplasts;

5-8: granulocrine secretion involving endoplasmic reticulum and Golgi apparatus, either alone or associated with plasmalemme.

\section{LES ODEURS}

Diverses odeurs sont émises au niveau des fleurs constituant des repères pour les pollinisateurs. Certaines d'entre elles nous sont agréables, d'autres désagréables. Les premières constituent les divers parfums dus à l'émission de terpènes et composés aromatiques volatiles. Les secondes résultent essentiellement de l'émission d'amines et d'indoles et sont destinées à attirer les insectes à larves nécrophages ou coprophages. 
Il est également intéressant de signaler qu'il existe quelques cas d'émission de substances semblables aux phéromones attirant les insectes mâles. HARBORNE (1977) analyse les divers travaux concernant les odeurs.

Dans la plupart des cas, les cellules sécrétrices sont éparses sur le périanthe. Parfois, elles sont groupées et portées par des organes floraux spécialisés appelés osmophores. On doit l'essentiel de la description de ces glandes à VoGEL (1962). Les osmophores sont essentiellement caractérisés par un épiderme glandulaire riche en substances de réserves et sont alimentés par le phloème.

\section{LES SÉCRÉTIONS DU PISTIL}

Elles se situent à divers niveaux : stigmate, style et ovule.

a) Le stigmate et la sécrétion stigmatique

Le stigmate correspond à la région du style recouverte de papilles. Sa position est soit terminale, soit latérale. On considère aujourd'hui qu'il existe deux grandes catégories de stigmates : des stigmates humides ("wet stigmas )) recouverts à l'anthèse d'un sécrétat liquide et des stigmates secs ("dry stigmas ») sans sécrétat liquide (voir HeSLOP-HARRISON et al., 1975 et DUMAS, 1976). Les espèces à stigmates secs appartiennent principalement à trois familles : Cruciferae, Caryophyllaceae et Compositeae.

HesLop-HARrison et ShivanNa (1977), à la suite d'une étude portant sur plus de 1000 espèces, estiment générale la sécrétion de protéines par les stigmates d'Angiospermes. Ces protéines de surface apparaissent très tôt dans le bouton floral. Chez les espèces à stigmates humides, ces protéines sont présentes avant même la sécrétion de l'exsudat liquide. Chez les espèces à stigmates secs, les protéines de surface forment une pellicule hydrophile extracuticulaire qui a été mise en évidence du fait de sa forte activité estérasique non spécifique (Fig. 4).

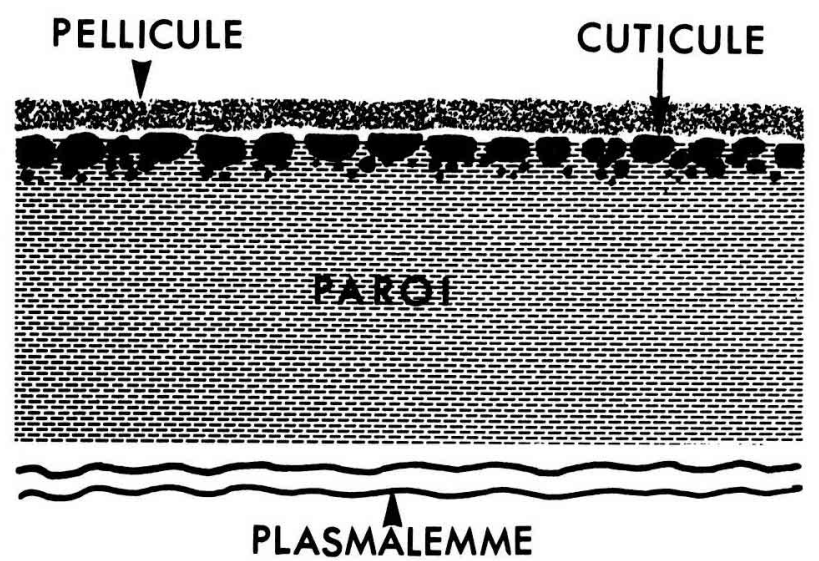

Fig. 4. - Coupe de la paroi externe d'une papille d'un stigmate de type sec

(d'après l'examen de Saponaria officinalis L.). Remarquer la discontinuité de la cuticule.

FIG. 4. - Section of the external wall of a papilla of a dry-type stigma (according to the examination of Saponaria officinalis L.). Note the discontinuity of cuticle.

Jusqu'à ces dernières années, les connaissances permettaient essentiellement de distinguer chez les stigmates humides, des sécrétions polysaccharidiques ou des sécrétions essentiellement lipophiles. Par exemple, chez Lilium, la sécrétion abondante est constituée en majeure partie de polysaccharides de type arabinogalactanes et d'acides uroniques (Aspinall et Rosell, 1978). Plus récemment, on s'est particulièrement intéressé à la présence de glycoconjugués. Ainsi, chez Gladiolus, KNox et al., (1976) ont isolé des glycoprotẻines et des glycolipides. Les proportions relatives entre protéines, sucres et lipides sont respecti- 
vement de 20, 23 et 0,1. Chez Brassica, RoBerTs et al., (1979) ont mis en évidence une glycoprotéine par électrofocalisation et GAUDE et Dumas (1980) ont révélé l'existence de glycoconjugués à l'aide d'une lectine marquée. Parmi les sucres, liés ou non, on remarque la faible proportion de mannose et de galactose (Clarke et al., 1979).

L'étude cytologique des processus de la sécrétion a été réalisée chez quelques espèces et tout récemment par Kristen (1977), Vithanage et Knox (1977), Dumas et al., (1978), Sedgley et Buttrose (1978), Tilton et HoRner (1980). Le type de sécrétion est tantôt eccrine, tantôt granulocrine. Mais, rares sont les études associant aux examens cytologiques, l'analyse chimique de l'exsudat. DumAs ( 1977 a et b) a montré que chez Forsythia l'exsudation essentiellement lipophile est le fait d'un mécanisme de type eccrine. Chez Aptenia, KRISTEN et al. (1979) ont observé des modifications du système endomembranaire au cours de la sécrétion de l'exsudat (sucres : $68 \%$, protéines : $8 \%$ ) le mécanisme étant alors essentiellement de type granulocrine.

b) Les sécrétions stylaires

Le style peut être soit compact ou plein, soit creux, c'est-à-dire muni d'un canal faisant communiquer la surface stigmatique et la cavité ovarienne (voir DumAs, 1976).

Comme le stigmate, le style est le siège de sécrétions. Les études d'ensemble sont rares (voir SEDGLEY et Buttrose, 1978). Gleeson et Clarke (1980) ont identifié des arabinogalactanes à la fois dans les stigmates et les styles de Lilium et Gladiolus.

De multiples travaux ont montré (voir LabarCa et Loewus, 1973 et Rosen, 1975) que le tube pollinique utilise des métabolites du pistil pour sa croissance, ce qui est confirmé par de récentes études autoradiographiques (Miki-Hirosige, 1981).

\section{c) Les sécrétions ovulaires}

Des sécrétions micropylaires sont connues chez les Angiospermes (voir : CHAO, 1977 b). ChaO (1977 a) a montré par cytochimie la nature pectique de l'appareil filiforme des synergides du sac embryonnaire (cellules de transfert).

\section{LES SÉCRÉTIONS POLLINIQUES ET TAPETALES}

Elles se conjuguent pour la mise en place de la paroi du grain de pollen singularisée par l'exine riche en sporopollénine enveloppée du manteau pollinique.

\section{a) La sécrétion de la paroi pollinique}

De nombreux travaux concernent l'ontogenèse de la paroi pollinique. HesLop-HARRISON et DickiNe son (1969) ont montré l'intervention du tapis staminal et de la microspore dans l'élaboration de la paroi pollinique. Les données récentes confirment le rôle du tapis qui produit des pro-orbicules de sporopollénine et certains constituants du manteau pollinique à partir du réticulum endoplasmique et des plastoglobules (Willemse et Reznickova, 1980; Reznickova et Willemse, 1980). Le dépôt majeur de sporopollénine de l'exine semble s'effectuer juste après la lyse de l'enveloppe callosique de la tétrade (REzNickova et al., 1980), puis, après destruction du tapis (DICKINSON et BELL, 1976). La sporopollénine pourrait s'incorporer par "accrétion" de globules et lamelles structurées analogues à des membranes (Fig. 5).

\section{b) Le manteau pollinique ou "pollen coat"}

Il a d'abord été connu sous forme d'un manteau huileux (" pollenkitl ") permettant l'agglomération des pollens entomogames. On y a également trouvé des substances hydrophiles (tryphine).

\section{Les substances lipophiles ou "pollenkitt"}

La nature précise des constituants lipidiques n'est pas connue. Leur rôle ne semble pas être exclusivement l'adhésion entre grains de pollen. En effet, malgré la présence du " pollenkitt " certains pollens sont pulvérulents. 


\section{TAPIS STAMINAL (2n)}

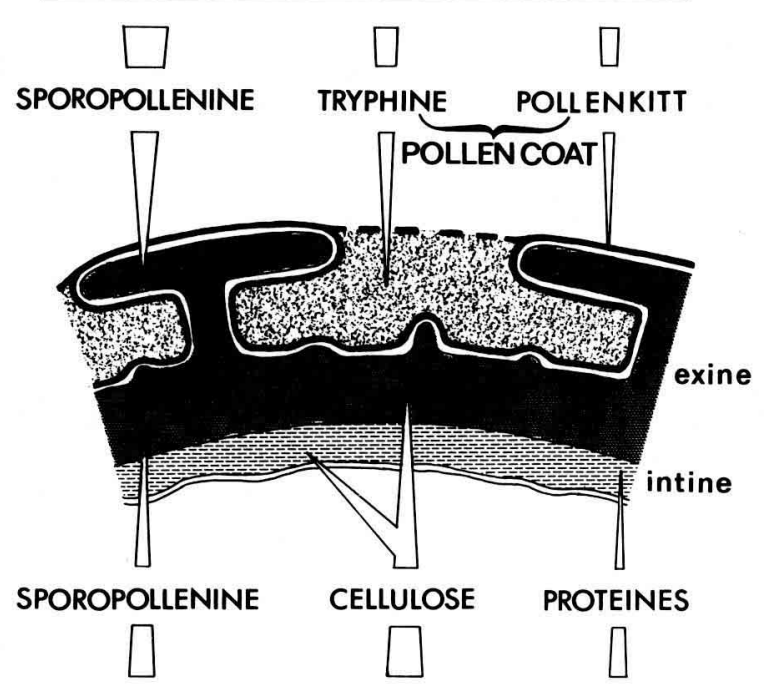

\section{MICROSPORE (n)}

FIG. 5. - Mise en place de la paroi et du manteau polliniques

et définition des domaines sporophytique et gamétophytique.

Il faut souligner que la sporopollénine, au début de la microsporogenèse a pour origine la microspore. Les dépôts suivants, les plus importants, sont d'origine tapétale. Des protéines d'origine diploïde (tapis) sont accumulées dans la tryphine. D'autres, localisées dans l'intine, sont d'origine gamétophytique (microspore).

Devant la confusion engendrée par la littérature à propos des termes "pollenkitt " et tryphine, nous suggérons dorénavant, de retenir uniquement le terme de manteau pollinique ou "pollen coat".

\section{FIG. 5. - Formation of the pollen wall and the pollen coat in their place} and definition of sporophytic and gametophytic areas.

It must be emphasized that sporopollenin at the beginning of microsporogenesis originates from the microspore. The next deposits, the most important, originate from the tapetum. Diploid originated proteins (tapis) are accumulated in the tryphin. Others, gametophytic originated, are located in intin.

Owing to the misunderstanding generated by literature concerning the terms "pollenkitt " and " tryphin ", we bereforth suggest to retain the single term of "pollen coat".

\section{Les substances hydrophiles ou tryphine}

Elles ont pour origine le tapis et pourraient intervenir dans les mécanismes d'incompatibilité et dans certaines allergies (KNox, 1979). Un examen plus précis des rôles de ces composés dans l'adhésion et la reconnaissance pollen-stigmate est envisagé par ailleurs (voir DUMAs et GAUDE, 1981 a).

Parmi les composés présents dans la tryphine, il y a de nombreuses protéines, enzymatiques (VITHA NAGE et KNOX, 1977; p. ex.) ou non (KNOX, 1979), certaines de ces protéines pouvant être des glycoprotéines à propriétés agglutinantes (Gaude et Dumas, 1980).

Les termes "pollenkitt " et tryphine ont été définis lors d'études déjà anciennes en microscopie photonique. Par la suite, une certaine confusion a été introduite dans la littérature, en particulier du fait de l'utilisation du terme “ pollenkitt " pour désigner l'ensemble du manteau pollinique. Nous pensons que le terme "pollen coat" doit être plus généralement utilisé. 
Cela permet de mieux saisir les travaux de $\operatorname{Hesse}(1979,1980$ a et b) chez qui nous rencontrons cette confusion de termes. Cet auteur apporte d'intéressantes précisions sur l'ultrastructure du manteau pollinique et ses rapports avec le mode de pollinisation. La propriété collante du manteau pollinique est liée à sa répartition plutôt qu'à son volume. Il présenterait une structure et une densité aux électrons homogènes chez les espèces entomogames. Chez les espèces anémogames, le manteau pollinique serait plus dense mais hétérogène voire même parfois absent. Des formes intermédiaires semblent, en outre, exister.

La présence de pigments pourrait guider les insectes pollinisateurs et agir comme écran protecteur contre les rayonnements U.V. (voir : STANLEY et LiNSKENS, 1974). Des huiles essentielles ou terpènes issus du tapis (" odeurs alimentaires" pour les pollinisateurs) semblent injectés dans les cryptes de l'exine au cours de la maturation finale du pollen.

\section{SÉCRÉTIONS ET FÉCONDATION}

Le rôle des sécrétions lors de la pollinisation est évident. Par contre, lors de la fécondation, les interventions de processus complexes et encore incomplètement connus, couplés à des sécrétions, demandent à être précisées. Ces interventions peuvent être groupées en deux types.

Le premier type concerne les mécanismes complexes de l'adhésion et de la reconnaissance pollen pistil, objets d'une étude détaillée (Dumas et Gaude, 1981 b).

Le second type concerne la croissance et le cheminement du tube pollinique dans le pistil. Le tube pollinique, depuis le stigmate jusqu'au sac embryonnaire, effectue un parcours complexe. L'hypothèse d'un chimiotropisme a été émise et de nombreuses substances comme le calcium, le bore, l'AMPc et autres hormones, etc., ont été testées. Aucune ne semble correspondre à l'agent chimiotropique universel (voir MAS $\leftarrow$ CARENHAS, 1975). Il est évident, par ailleurs, que des voies de pénétration sont tracées dans le pistil (voir Dumas et al., 1978) guidant la progression du tube pollinique. Des microtubules et microfilaments semblent orienter les flux de vésicules à l'apex du tube pollinique (voir Mascarenhas, 1975). Des mouvements d'ions potassium et calcium liés à une dépolarisation des membranes (HERTH, 1978; p. ex.) ainsi que l'exocytose polarisée des vésicules golgiennes, peut être sous l'action d'un signal chimiotropique (hypothèse personnelle), pourraient expliquer les brusques changements de direction du tube pollinique.

Le tube pollinique utilise des métabolites du pistil pour édifier sa paroi (LABARCA et LoEwus, 1973). Cette notion établie chez les espèces à style creux semble pouvoir être généralisée aux espèces à style plein, du fait de la nature pectique des parois du tissu de transmission (LinsKens, 1974 et MiKi-Hirosige, 1981).

En conclusion, une succession de processus sécrétoires interviennent tout au long du cheminement du gamète mâle vers le gamète femelle chez les Angiospermes. Ils se manifestent au cours de deux phases distinctes. La première phase est celle au cours de laquelle le gamétophyte mâle, c'est-à-dire le grain de pollen, est transporté depuis l'anthère jusqu'au stigmate. La seconde phase consiste dans le développement du tube pollinique et son accès au sac embryonnaire.

Ces processus sécrétoires sont évidemment pro parte bénéfiques pour l'animal qui joue dans bien des cas le rôle de vecteur du pollen et pour les Apidés, en particulier. Mais, leur finalité, pour être saisie dans son intégralité, doit être replacée dans le contexte de la reproduction sexuée des plantes. Elle est alors incontestablement de favoriser à tous les niveaux la fécondation croisée et parfois, en cas d'échec, l'autofécondation.

La grande diversité des sécrétions impliquées a entrainé une étude particulière de chaque type. Certains processus sont aujourd'hui partiellement élucidés. L'étude de certains autres en est encore à ses premiers balbutiements. Les recherches actuelles portent particulièrement sur les phénomènes qui concourent à la mise en place, au niveau du pollen d'une part et du stigmate d'autre part, de substances de surface assurant les contacts puis les reconnaissances et le choix génétique du gamète mâle par le gamétophyte et le gamète femelle. Ces phénomènes paraissent revêtir une telle importance qu'ils relèguent à la seconde place les sécrétions intervenant d'une manière moins structurée dans le transport du pollen (revue in: DUMAS et GAUDE, 1981 b). Cette conception devrait marquer la recherche en Biologie florale des années à venir et les résultats des investigations en ce domaine devraient être la source de nouvelles conceptions tant dans la recherche fondamentale qu'appliquée.

Reçu pour publication en février 1981. Eingegangen in Februar 1981. 


\section{ZUS AMMENFASSUNG}

\section{SEKRETIONEN UND BIOLOGIE DER BLÜTE. I. ART UND BEDEUTUNG DER SEKRETIONEN} BEI DER BESTÄUBUNG UND DER BEFRUCHTUNG. NEUERE ERGEBNISSE

Im Verlaufe der letzten zehn Jahre wurden die morphologischen Aspekte der Blütenbiologie durch Ergebnisse mit vorwiegend dynamischen Gesichtspunkten ergänzt. Die Rolle der Sekretionen in den verschiedenen Phasen der Bestäubung und der Befruchtung sowie die Mechanismen dieser Sekretionen wurden teilweise aufgeklärt.

Diese Zusammenstellung bringt die neuesten Resultate über den Nektar, den Duft und die Sekretionen des Tapetum, des Pollens und des Pistills.

Die Zusammensetzung des Nektars wurde unter Benutzung moderner Techniken der Analyse von Zuckern und Aminosäuren (insbesondere durch GC) genauer bestimmt. Die letzteren besitzen eine bemerkenswerte Spezifität und können mit bestimmten Mineral-Ionen dem Nektar attraktive Eigenschaften verleihen, welche das Verhalten der Tiere bei der Bestäubung lenken.

Mehrere cytologische und cytochemische Untersuchungen, die mit Hilfe der TransmissionsElektronenmikroskopie durchgeführt worden sind, haben wenigstens zum Teil die Sekretionsmechanismen aufgeklärt.

Die Produktion von Düften durch die Zellen des Perianths oder durch die Osmophoren, welche die Bestäuber anlocken, scheint vorwiegend auf der Emission von aromatischen Substanzen vom Typ der Terpene oder von Aminen zu beruhen.

Die Sekretionen des Stempels erfolgen auf verschiedenen Niveaus : An der Narbe (Stigma), das die Aufnahme des Pollenkorns gewährleistet, am Griffel, in dem der Pollenschlauch wandert, und an der Samenanlage. Die Narben werden in zwei Kategorien klassifiziert, feuchte und trockene, entsprechend der Menge der Sekrete.

Die Proteine in den Exsudaten des Stigma scheinen bei allen Narbe generell vorzukommen. Diese Proteine wurden durch die Intensität ihrer nichtspezifischen Esterase-Aktivität nachgewiesen. Eine zytologische und zytochemische Untersuchung des Sekretionsprozesses wurde in Angriff genommen, aber die Resultate sind bisher noch unvollständig.

Die neuen Ergebnisse führen zu einer klaren Beschreibung der Art und der Bedeutung der Sekrete des Stempels und der Samenanlage, die in das Wachstum und in die Wanderung des Pollenschlauches eingreifen.

Wahrscheinlich haben im Laufe der letzten Jahre diejenigen Arbeiten über die Sekrete die interessantesten Informationen geliefert, die aus der Pollenhülle, dem " pollen coat ", stammen (Proteine, Glykoproteine, Lipide, Pigmente...).

Diese Substanzen gewährleisten die Adhäsion der Pollenkörner untereinander, vor allem aber sind sie, zusammen mit den Sekreten der Narbe für das Phänomen der Anheftung und der gegenseitigen "Erkennung" von Pollen und Stigma (Narbe) verantwortlich. Diese Phänomene entscheiden über die Kompatibilität zwischen den beiden Partnern, eine unverzichtbare Voraussetzung dafür, dass aus der Bestäubung eine erfolgreiche Befruchtung wird.

Heute ist das Hauptinteresse der Forschung auf die zellulären und molekularen Wechselwirkungen konzentriert, die während der Bestäubung zwischen den beiden Partnern stattfinden und die für die sexuelle Fortpflanzung verantwortlich sind: Der Pollen und das Pistill.

\section{SUMMARY}

SECRETIONS AND FLORAL BIOLOGY. I. NATURE AND PART OF SECRETIONS IN POLLINATION AND FERTILIZATION. LATE DATA

During the last ten years, morphological aspects related to floral biology have been supplemented by more dynamic aspects. Parts played by secretions in the different phases of pollination and fertilization processes, as well as mechanisms of these secretions, were partly cleared up. 
This review the latest results dealing with nectars fragances, pollen, tapetum and pistil secretions.

Composition of nectars was determined by modern techniques of analysis of sugars and amino acids (especially GC). The latter show a remarkable specificity and can, with certain mineral ions, confer attractive qualities on nectar and thus guide the choice of pollinators.

Several cytological and cytochemical studies by means of electron microscope permitted to clear up mechanisms of secretion, at least partly.

Production of pollinators-attracting fragrances by cells of perianth orf by osmophors seems to be mainly due to the emission of aromatic substances of the terpene or amine type.

Pistil secretions take place at different levels : the stigma, that receives the pollen grain, the style where the pollen tube progresses and the ovule. Stigma are classified in two groups, wet and dry, according to the abundance of secretions. It seems to be possible to generalize the presence of proteins in the stigma exudate to all stigmas. These proteins were detected by their high non-specific esterase activity. The cytological and cytochemical study of the secretion process was approached but results are still incomplete.

Recent data permit the nature and part of style and ovule secretions that interfere in growth and progress of the pollen tube to be specified.

It is probably the research on secretion products located in the pollen wall and the formation of pollen coat (proteins, glycoproteins, lipids, pigments) that gave the most interesting informations during the recent years. Indeed these substances ensure the possible adhesion of pollen grains among them but, jointly with stigma secretions, they are responsible overall for pollen-stigma sticking and recognition phenomena. These phenomena manage the compatibility between both partners which is absolutely necessary in order that pollination results an efficient fertilization.

The primary research is now centred on cellular and molecular interactions that occur during pollination between both partners responsible for sexual reproduction : pollen and pistil.

\section{BIBLIOGRAPHIE}

Aspinall G. O. et Rosell K. G., 1978. - Polysaccharide component in the stigmatic exudate from Lilium longiflorum. Phytochem., 17, 919-922.

Baker D. A., HALl J. L. et Thorpe J. R., 1978. - A study of the extrafloral nectaries of Ricinus communis. New Phytol., 81, 129-137.

BaKer H. G., 1977. - Non sugar chemical constituents of nectar. Apidologie, 8, 349-356.

Barker R. J., Lehner Y. et Kunzmann M. R., 1980. - Pesticides and honey bees : nectar and pollen contamination in Alfalfa treated with dimethoate. Arch. Environm. Contam. Toxicol., 9, 125-133.

BENNER U. et SCHNEPF E., 1975. - Die Morphologie der Nektarausscheidung bei Bromeliaceen : Beteiligung des Golgi Apparates. Protoplasma, 85, 337-349.

BENTLEY B. L., 1977. - Extrafloral nectaries and protection by pugnacious bodyguards. Ann. Rev. Ecol. Syst., 8, 407-437.

CHAO C. Y., 1977 a. - Light microscopy detection of P.A.S. - Positive substances with thiosemicarbazide in freeze substituted ovaries of Paspalum longiflorum before pollination. Histochem., 54, 159-168.

ChaO C. Y., 1977 b. - Further cytological studies of a periodic acid schiff's substance in the ovules of Paspalum orbiculare and P. longiflorum. A mer. J. Bot., 64, 921-930.

Clarke A. E., Anderson R. L. et Stone B. A., 1979. - Form and function of arabinogalactans and arabinogalactan-proteins. Phytochem., 18, 521-540.

Dickinson H. G. et BELl P. R., 1976. - The changes in the tapetum of Pinus banksiana accompanying formation and maturation of the pollen. Ann. Bot., 40, 1101-1109.

Dumas C., 1976. - Stigmates sécréteurs et lipides neutres sécrétés. Actualités Botaniques (1978), 75, 6168. 
Dumas C., 1977 a. - Établissement d'un modèle de la cinétique de la sécrétion lipo-polyphénolique du stigmate de Forsythia intermedia Zabel. C. R. Acad. Sci. (Paris), 284, 1777-1779.

Dumas C., 1977 b. - Lipochemistry of the progamic stage of a self-incompatible species : Neutral lipids and fatty acids of the secretory stigma during its glandular activity, and of the solid style, the ovary and the anther in Forsythia intermedia Zabel (heterostylic species). Planta, 137, 177-184.

Dumas C. et Gaude T., 1981 a. - Stigma-pollen recognition : a new look. In : Internat. Sympos. Adv. in Plant Cytoembryology (Lublin, juin 1980). Acta Soc. Bot. Pol., 50 (sous presse).

Dumas C. et Gaude T., 1981 b. - Sécrétions et Biologie florale : II. Leurs rôles dans l'adhésion et la reconnaissance pollen-stigmate. Données récentes, hypothèses et notion d'immunité végétale. A ctualités Botaniques (sous presse).

Dumas C., Rougier M., Zandonella P., Ciampolini F., Cresti M. et Pacini E., 1978. - The secretory stigma in Lycopersicum peruvianum Mill. Ontogenesis and glandular activity. Protoplasma, 96, 173187.

Eriksson M., 1977. - The ultrastructure of the nectary of Red clover (Trifolium pratense). J. Apicult. Res., 16, 184-193.

Faegri K. et PiJl, L. van der, 1971. - The principles of pollination ecology ( 2 nd ed.). Pergamon Press, Oxford.

FAHN A., 1979 a. Ultrastructure of nectaries in relation to nectar secretion. Amer. J. Bot., 66, 977-985.

FAHN A., 1979 b. - Secretory tissues in Plants. Academic Press, New York, San Francisco.

FAHN A. et Benouaiche P., 1979. - Ultrastructure of the nectary of Musa paradisiaca L. var. sapientum kuntze and its relation to nectar secretion. Ann. Bot., 44, 85-93.

FAHN A. et RACHMILEvitz T., 1970. - Ultrastructure and nectar secretion in Lonicera japonica. Bot. $J$. Linn. Soc., 63, suppl., 51-56.

FAHN A. et RACHMILEvitz T., 1975. - An autoradiological study of nectar secretion in Lonicera japonica Thunb. Ann. Bot., 39, 975-976.

Findlay N. et Mercer F. V., 1971. - Nectar production in Abutilon. II. Submicroscopic structure of the nectary. Austral. J. Biol. Sci., 24, 657-664.

FRANKel R. et Galun E., 1977. - Pollination mechanisms, reproduction and plant breeding. SpringerVerlag. Berlin, Heidelberg, New York.

Gaude T. et Dumas C., 1980. - Des glycoconjugués sont-ils impliqués dans l'adhésion pollen-stigmate? Communication Congrès Soc. Chimie Biol., G.V. (Lille, sept. 1980).

Gleeson P. A. et Clarke A. E., 1980. - Comparison of the structures of the major components of the stigma and style secretions by Gladiolus : the arabino-3, 6-galactans. Carbohydrate Res., 83, 187 192.

GuNNiNG B. E. S. et Hughes J. E., 1976. - Quantitative assessment of symplastic transport of pre-nectar into the trichomes of Abutilon nectaries. Aust. J. Pl. Physiol., 3, 619-637.

HARBORNE J. B., 1977. - Biochemistry of plant pollination pp. 28-57. In : Introduction to ecological biochemistry. Academic Press; Londres, San Francisco, New York.

HeINRICH G., 1975. - Über den glucose-Metabolismus in Nektarien zweir Aloe-Arten und über den Mechanismus der Pronektar-Sekretion. Protoplasma, 85, 351-371.

HERTH W., 1978. - Ionophore A 23187 stops tip growth, but not cytoplasmic streaming in pollen tubes of Lilium longiflorum. Protoplasma, 96, 275-282.

Heslop-Harrison J. et Dickinson H.G., 1969. - Time relationships of sporopollenin synthesis associated with tapetum and microspores in Lilium. Planta, 84, 199-214.

Heslop-Harrison J., Heslop-Harrison Y. et Barber J., 1975. - The stigma surface in incompatibility response. Proc. R. Soc., 188, 287-297.

Heslop-Harrison Y. et Shivanna K. R., 1977. - The receptive surface of the angiosperm stigma. Ann. Bot., 41, 1233-1258. 
Hesse M., 1979. - Development and ultrastructure of pollenkitt and exine in closely related entomophilous and anemophilous angiosperms. Salicaceae, Tiliacecae, and Ericaceae. Flora, 168, 540-557.

Hesse M., 1980 a. - On the attachment of pollen on flower visiting insects by pollenkitt and Viscin threads. Pl. Syst. Evol., 123, 135-148.

Hesse M., 1980 b. - Development and ultrastructure of pollenkitt and exine in closely related entomo and anemophilous angiosperms: Alismataceae, Liliaceae, Juncaceae, Cyperaceae, Poaceae, A raceae. Pl. Syst. Evol., 134, 229-267.

Knox R. B., 1979. - Pollen and allergy. The Institute of Biology's Studies in Biology., 107, Edward Arnold ed. Londres.

Knox R. B., Clarke A. E., Harrison S., Smith P. et Marchalonis J. J., 1976. - Cell recognition in plants : Determinants of the stigma surface and their pollen interactions. Proc. Natl. Acad. Sci., U.S.A., 73, 2788-2792.

KrISTEN U., 1977. - Granulocrine Ausscheidung von Narbensekret durch Vesikel des Endoplasmatischer retikulums bei Aptenia cordifolia. Protoplasma, 92, 243-251.

Kristen U., Biederman M., Liebezeit G., Dawson R. et BöHN L., 1979. - The composition of stigmatic exudate and the ultrastructure of the stigma papillae in Aptenia cordifolia. European J. Cell Biol., 19, 281-287.

LABARCA C. et LoEwus F., 1973. - The nutritional rôle of pistil exudate in pollen tube wall formation in Lilium longiflorum. II. Production and utilization of exudate from stigma and stylar canal. Plant Physiol., 52, 87-92.

Linskens H. F., 1974. - Fertilization in Higher Plants. North-Holland Publ. Co. Amsterdam. Oxford.

LÜTTGE U., 1977. - Nectar composition and membrane transport of sugars and amino acids : a review on the present state of nectar research. Apidologie, 8, 305-319.

LÜTtGe U. et SchNPEF E., 1976. - Elimination processes by glands. Organic substances. In : Transport in Plants. II. Encyclopedia of Plant Physiology, 2, Part. 8, pp. 244-277. Springer Verlag. Berlin.

Mascarenhas J. P., 1975. - The biochemistry of angiosperm pollen development. Bot. Rev., 41, 259314.

Miki-Hirosige H., 1981. - $\alpha$-d glucose, myoinositol and pectin-units in transmitting tissue. In : Acta Soc. Bot. Pol., 50 (sous presse).

Pais M. S., Chaves Das Neves H. J., 1980. - Sugar content of the nectary exudate of Epipactis atropurpurea Rafin. Apidologie, 11, 39-45.

PIJL L. van der, 1954. - Xylocopa and flowers in tropics. II. Observations on Thunbergia, Ipomoea, Costus, Centrosema and Canavalia. K. Nederlandse ak. Wetens. Proc., 57, 541-551.

RACHMILEvitz T. et FAHN A., 1973. - Ultrastructure of nectaries of Vinca rosea L., Vinca major L. and Citrus sinensis Osbeck cv. Valencia and its relation to the mechanism of nectar secretion. Ann. Bot., 37, 1-9.

Reznickova S. A., VAN Aelst A. C. et Willemse M. T. M., 1980. - Investigation of exine and orbicule formation in the Lilium anther by SEM. Acta bot. Neerl., 29, 157-164.

Reznickova S. A., Willemse M. T. M., 1980. - Formation of pollen in the anther of Lilium. II. The function of the surrounding tissues in the formation of pollen and pollen wall. Acta bot. Neerl., 29, 141-156.

Roberts I. N., Stead A. D., Ockendon D. J. et Dickinson H. G., 1979. - A glycoprotein associated with the acquisition of the self-incompatibility system by maturing stigmas of Brassica oleracea. Planta, 146, 179-183.

Rosen W. G., 1975. - Pollen pistil interactions. In : The Biology of the male gamete, DuCKett J. G. et Racey P. A. ed., pp. 153-164. Academic Press, Londres. New York.

SChNePf E., 1977. - Bau und Feinbau der Naktarien und der Mechanismus der Nektarsekretion. Apidologie, 8, 295-304. 
SCHNEPF E. et Pross E., 1976. - Differentiation and redifferentiation of a transfer cell : Development of septal nectaries of Aloe and Gasteria. Protoplasma, 89, 105-115.

Sedgley M. et Buttrose M. S., 1978. - Structure of the stigma and style of the avocado. Austral. J. Bot., 26, 663-682.

Stanley R. G. et Linskens H. F., 1974. - Pollen. Biology, Biochemistry and Management. Springer Verlag. Berlin, Heidelberg, New York.

Tilton V. R., Horner H. T., 1980. - Stigma, style and obtulator of Ornithogalum caudatum (Liliaceae) and their function in the reproductive process. Amer. J. Bot., 67, 1113-1131.

VAsilujev A. E., 1969. - (en russe) Submicroscopic morphology of nectary cells and problems of nectar secretion. Bot. J. SSSR, 54, 1015-1031.

VAsilijev A. E., 1971. - (en russe) New data on the ultrastructure of the cells of flower nectary. Bot. $J$. SSSR., 56, 1292-1306.

Vithanage H. IM. V. et KNox R. B., 1977. - Development and cytochemistry of stigma surface and responce to self - and foreign - pollinations in Helianthus annuus. Phytomorphology, 27, 168-179.

Vogel S., 1962. - Duftdrüssen im Diemst der Bestäuburg. Uber Bau und Funktion der Osmophoren. Akad. Wiss. Lit. Mainz, Abb. Math. naturwiss. kl., 10, 598-763.

VoGeL S., 1974. - Ölblumen und ölsammelnde Bienen in: Tropische und subtropische Pflanzenwelt. Akad. Wiss. Lit. Franz Steiner Verlag. Wiesbaden.

Wergin P. W., Elmore C. D., HANNy B. W. et INGBER B., 1975. - Ultrastructure of the subglandular cells from the foliar nectaries of cotton in relation to the distribution of plasmodesmata and symplastic transport of nectar. Amer. J. Bot., 62, 842-849.

Willemse M. T. M. et Reznickova S. A., 1980. - Formation of pollen in the anther of Lilium. I. Development of the pollen wall. Acta Bot. Neerl., 29, 127-140.

Zandonella P., 1970. - Infrastructure des cellules du tissu nectarigène floral de quelques Caryophyllaceae. C.R. Acad. Sci. (Paris), 270, 1310-1313.

Zandonella P., 1972. - Le nectaire floral des Centrospermales. Localisation, morphologie, anatomie, histologie, cytologie. Thèse, Lyon.

Zandonella P., Piolat C., 1981. - Nectaires et sécrétion du nectar chez Thunbergia laurifolia Lindl. (Acanthaceae). Actualités botaniques (sous presse).

Zauralov O. A. et Pavlinova O. A., 1975. - Transport and conversion of sugars in nectaries in connection with the secretory function. Fiziol. Rastenii, 22, 500-507. 